\title{
Aprendizaje divertido de programación con Gamificación
}

\author{
Jefferson Beltrán Morales ${ }^{1}$, Héctor Sánchez ${ }^{2}$, Mercedes Rico ${ }^{2}$ \\ jtbeltran@uce.edu.es; sasah@unex.es; mricogar@unex.es \\ ${ }^{1}$ Facultad de Ingeniería y Ciencias Aplicadas, Universidad Central del Ecuador, Quito, Ecuador. \\ ${ }^{2}$ Centro Universitario de Mérida, Universidad de Extremadura, Mérida, España.
}

DOI: 10.17013/risti.41.17-33

\begin{abstract}
Resumen: Aplicar estrategias de gamificación implementadas en una plataforma virtual de aprendizaje como apoyo a clases presenciales, es una propuesta educativa que incrementa la motivación y compromiso en la realización de actividades y tareas autónomas haciendo divertido el aprendizaje. Esta propuesta se aplicó en la asignatura Programación I (P-I) de la Facultad de Ingeniería y Ciencias Aplicadas (FICA) de la Universidad Central de Ecuador (UCE), donde se evaluó el rendimiento académico de los estudiantes comparando grupos: experimental (metodología gamificada) y de control (metodología tradicional). Mediante encuestas también se valoró el curso gamificado y las estrategias empleadas que más aportaron a hacer divertido el aprendizaje. Como conclusión de la investigación se tiene que una plataforma virtual de aprendizaje gamificada hizo que los alumnos se diviertan aprendiendo a programar influyendo positivamente en su motivación por el desarrollo de actividades autónomas de aprendizaje, además que también permitió mejorar su rendimiento académico.
\end{abstract}

Palabras-clave: Gamificación; e-Learning; Programación; Motivación; Actividades de aprendizaje.

\section{Fun programming learning with Gamification}

\begin{abstract}
Applying gamification strategies implemented in a virtual learning platform as support for face-to-face classes, is an educational proposal that increases motivation and commitment in carrying out autonomous activities and tasks, making learning fun. This proposal was applied in the Programming I (PI) subject of the Faculty of Engineering and Applied Sciences (FEAS) of the Central University of Ecuador (CUE), where the academic performance of the students was evaluated by comparing groups: experimental (gamified methodology) and control (traditional methodology). Surveys also assessed the gamified course and the strategies used that contributed the most to making learning fun. As a conclusion of the research, a gamified virtual learning platform made the students have fun learning to program, positively influencing their motivation for the development of autonomous learning activities, in addition to improving their academic performance.
\end{abstract}


Keywords: Gamification; e-Learning; Programming; Motivation; Learning activities.

\section{Introducción}

Uno de los principales problemas que enfrentan los docentes es cómo conseguir de sus estudiantes una motivación adecuada y el compromiso en el proceso de aprendizaje. (Mas \& Medinas, 2007). Las asignaturas relacionadas a la programación no están fuera de esta problemática, los profesores de programación observan que, en general, los alumnos de primer y segundo semestres muestran algunos rasgos que dificultan el aprendizaje, tales como: falta de implicación estudiantil y apatía por la programación (Oviedo \& Ortiz Uribe, 2012). La programación requiere de mucha práctica, y que para mantenerla los estudiantes deben estar adecuadamente motivados (Jenkins, 2001) (Fuentes-Rosado \& Moo-Medina, 2017). Los alumnos prefieren más la práctica que la teoría, pero no les gusta las actividades de aprendizaje autónomas, mostrando una falta de motivación por el autoaprendizaje (Beltrán, Sánchez, \& Rico, Análisis cuantitativo y cualitativo del aprendizaje de Programación I en la Universidad Central del Ecuador, 2015).

El docente debe entonces incidir sobre el aprendizaje de sus alumnos a través del desarrollo de la motivación por el estudio de programación; proponiendo estrategias e implementando métodos, herramientas y recursos que favorezcan la motivación intrínseca para el estudio, creando un espacio emocionante y agradable que fomente la participación y el interés por la programación y por las tareas fuera del aula (González C \& Mora, 2015).

Los avances de las Tecnologías de la Información y Comunicaciones (TIC) a través de los entornos virtuales de aprendizaje (EVA), aportan instrumentos para el desarrollo del proceso educativo de una manera más integrada, que junto a estrategias de Gamificación ayudan a promover la motivación en el desarrollo de las actividades de aprendizaje, se estimula la participación activa de los estudiantes, genera un proceso de retroalimentación entre los compañeros de estudio, se crea confianza entre los alumnos y convierte las actividades difíciles en procesos más sencillos (Mas \& Medinas, 2007) (Lorenzo \& Buendía, 2016).

En educación, la gamificación se aplica desarrollando actividades para lograr ciertos objetivos de aprendizaje, los estudiantes participan en un entorno divertido y competitivo aumentando de esta manera su motivación (Kyriakova, Angelova, \& Yordanova, 2014). La gamificación implementada en un entorno e-Learning, influye en la mejora del rendimiento de los estudiantes, principalmente en el desarrollo de sus tareas en ambientes virtuales, y en menor medida en las calificaciones (Ibáñez, Di-Serio, \& Delgado-Kloos, 2014) (Urh, Vukovic, Jereb, \& Pintar, 2015).

La presente investigación presenta los resultados de aplicar la metodología de Aprendizaje Gamificada propuesta dentro un entorno e-Learning, con el objetivo de incrementar la motivación y el compromiso en el desarrollo de tareas autónomas de aprendizaje en la asignatura P-I de la FICA de la UCE. Se describe el marco teórico, la metodología de investigación usada, luego se detallan los resultados obtenidos, y finalmente se listan las conclusiones y se proponen futuros trabajos de investigación. 


\section{Marco teórico}

La gamificación es "el empleo de mecánicas de juego en entornos y aplicaciones no lúdicos, con el fin de potenciar la motivación, la concentración, el esfuerzo, la fidelización y otros valores positivos comunes a todos los juegos" (González C \& Mora, 2015). Mediante la aplicación de ciertos elementos lúdicos, como insignias, puntos, tableros, etc., los jugadores incrementan su tiempo de participación en el juego, colaboran más y sobre todo que aprenden gracias a la información que reciben durante su estancia en el mismo. De esta forma se consigue que el jugador adquiera conocimientos de los que carecía, haciendo importante su uso para fines educativos.

En educación, la gamificación es "el uso de los elementos de juego (dinámicas, mecánicas y componentes) en un ambiente de aprendizaje, generalmente apoyado en las TIC" (Simões, Díaz, \& Fernández, 2013). Las técnicas usadas en la gamificación se transforman en estrategias útiles para aplicarlas en la enseñanza, contribuyendo a la motivación de los alumnos hacia el aprendizaje. Es este contexto, la gamificación trata de sistematizar un procedimiento a la hora de impartir las clases, o en la realización de actividades innovadoras y divertidas de aprendizaje (Ibáñez, Di-Serio, \& Delgado-Kloos, 2014), aumentando su interés y grado de atención, la actividad investigadora y la adquisición y consolidación de conocimientos (Serna, Mauricio, San Miguel, \& Megías, 2016) (Pérez \& Almela, 2018). Estas actividades pueden ser autónomas, es decir, tareas que por sí solo puede realizar el alumno con una adecuada guía del docente (aprender a aprender).

Una de las plataformas virtuales más usadas como apoyo al proceso de aprendizaje es Moodle, pues posee varias características, funcionalidades y plugins que permiten implementar fácil y efectivamente las dinámicas y mecánicas de juego, como: puntos, insignias, tableros, avatares, barras de progreso, etc. (Cacho \& Rodrigo, 2014).

Varios investigadores proponen métodos donde el estudiante aprende a programar completando tareas de programación (codificación y ejecución de código) con retroalimentación correctiva dentro de una interfaz e-Learning basada en el juego (Watson, Li, \& Lau, 2011) (Guo, Chai, \& Qian, 2010). A diferencia del aprendizaje tradicionales, este concepto apoyado en las dinámicas y mecánicas de juego que ofrece la gamificación, desafíos, niveles de juego y experiencia e-Learning (Schefer-Wenzl \& Miladinovic, 2018); permite un aprendizaje divertido, desarrollando rápidamente en los alumnos una comprensión de los conceptos de programación a través de un conjunto de ejemplos, además de fomentar la motivación, colaboración, participación, trabajo en equipo y el compromiso en actividades de desarrollo de software (MachucaVillegas, Gasca-Hurtado, Restrepo, \& Puente, 2020) (Gasca-Hurtado, Gómez-Álvarez, \& Manrique-Losada, 2019).

\section{Desarrollo Experimental - Metodología}

\subsection{Contexto}

La investigación se realizó en la asignatura presencial P-I en la FICA de la UCE entre los años 2015 y 2019 en 9 cursos (S1, S2, S3, S4, S5, S6-1, S6-2, S7-1, S7-29). P-I se imparte en el primer semestre de las Carreras de Ingenierías: Informática, Matemática, Computación 
Gráfica, Diseño Industrial, Computación y Sistemas de Información, periodo dividido en dos hemi-semestres, en el primero ( $\mathrm{I} \mathrm{H}-\mathrm{S}$ ) el estudiante adquiere los conocimientos iniciales para diseñar algoritmos usando pseudocódigo y diagramas de flujo, para en el segundo hemi-semestre (II H-S) aprender los fundamentos de programación en Java utilizando las diferentes técnicas algorítmicas de la programación estructurada y de la programación orientada a objetos (clases, objetos, atributos y métodos).

\subsection{Hipótesis y Variables}

- Hipótesis: La implementación de estrategias de gamificación sobre una plataforma virtual de aprendizaje para desarrollar tareas autónomas influye sobre la motivación de los estudiantes y contribuye en mejorar su rendimiento académico, en la asignatura P-I de la FICA de la UCE.

- Variable independiente: Estrategias de gamificación.

- Variables dependientes:

- Motivación.

- Rendimiento académico.

\subsection{Diseño de la Investigación y Muestra}

Las principales actividades de la investigación se muestran en la Figura 1:

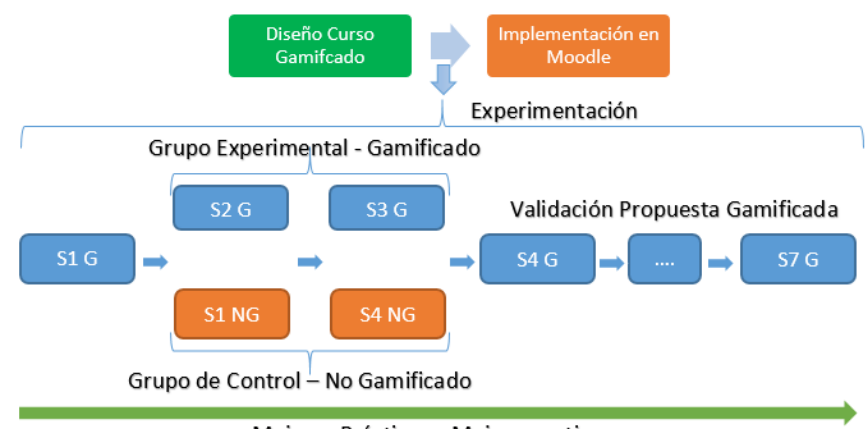

Mejores Prácticas - Mejora continua

Figura 1 - Diseño de la investigación

Con un enfoque cuantitativo, para medir los resultados del Aprendizaje Gamificado sobre la motivación y el compromiso de los estudiantes en el desarrollo de tareas autónomas y el rendimiento académico, se realizó un estudio con alcance explicativo, con un diseño cuasi-experimental con grupos de control (metodología normal) y experimental (metodología gamificada), en el que se emplean grupos intactos de comparaciones ya existentes, no equivalentes (Tejedor, 2000).

En el I H-S de todos los periodos se usó la metodología tradicional o normal. Inicialmente, se analizó en forma cuantitativa las notas finales en los S1, S2 y S3. En el II H-S del S1 se aplicó la metodología gamificada para todos los estudiantes; en cambio, en el S2 se crearon dos grupos en forma aleatoria para aplicar las dos metodologías por separado. 
Luego, en el II H-S del S3, se usaron las metodologías normal y gamificada en diferentes carreras. En cada experiencia se aplicaron mejoras metodológicas y tecnológicas a partir de la evaluación de la experiencia anterior. Cabe señalar que; en los dos grupos, control y experimental, el sílabo y los exámenes fueron comunes. Finalmente, en los siguientes semestres para validar la metodología propuesta, en el II H-S se utilizó la metodología gamificada. En la Tabla 1, se muestra la cantidad de estudiantes por metodología en cada semestre.

Además, con un enfoque cualitativo se aplicaron encuestas electrónicas anónimas a los estudiantes que terminaron el curso gamificado con el objetivo de valorar cuáles estrategias de gamificación aplicadas motivan, incentivan y comprometen a los estudiantes en el aprendizaje de P-I y permiten obtener mejores calificaciones. La cantidad de alumnos que responden la encuesta se listan en la Tabla 1.

\begin{tabular}{|c|c|c|c|c|c|}
\hline Semestre & Metodología & $\begin{array}{l}\text { Carrera } \\
\text { Ingeniería }\end{array}$ & Total & II H-S & $\begin{array}{l}\text { Responde } \\
\text { Encuesta }\end{array}$ \\
\hline$S 1$ & Gamificada & $\begin{array}{l}\text { Computación } \\
\text { Gráfica }\end{array}$ & 17 & 10 & 9 \\
\hline$S_{2}$ & Normal & Matemática & 12 & 6 & - \\
\hline$S 2$ & Gamificada & Matemática & 13 & 10 & 10 \\
\hline$S_{3}$ & Normal & Diseño Industrial & 31 & 28 & - \\
\hline$S_{3}$ & Gamificada & Matemática & 33 & 32 & 30 \\
\hline$S_{4}$ & Gamificada & Informática & 23 & 22 & 19 \\
\hline$S_{5}$ & Gamificada & Informática & 7 & 6 & 7 \\
\hline$S 6-1$ & Gamificada & \multirow{2}{*}{$\begin{array}{l}\text { Sistemas de } \\
\text { Información }\end{array}$} & 31 & 31 & \multirow{2}{*}{44} \\
\hline$S 6-2$ & Gamificada & & 45 & 43 & \\
\hline$S_{7-1}$ & Gamificada & \multirow{2}{*}{$\begin{array}{l}\text { Sistemas de } \\
\text { Información }\end{array}$} & 40 & 39 & \multirow{2}{*}{44} \\
\hline$S_{7-2}$ & Gamificada & & 43 & 43 & \\
\hline Total & & & 295 & 270 & 163 \\
\hline
\end{tabular}

Tabla 1 - Cantidad de estudiantes, semestres y metodología usada

\subsection{Análisis de datos}

En el análisis cualitativo las encuestas se presentan en tablas y gráficos de frecuencia y contingencia, utilizando para su descripción estadísticos tales como frecuencia absoluta y porcentajes.

Para medir los resultados en el estudio cuantitativo los datos se organizan en tablas de frecuencia, contingencia y figuras, utilizando estadígrafos descriptivos como porcentajes y frecuencia absoluta.

Para comprobar la hipótesis se hace un análisis estadístico con test referidos a la variable "rendimiento académico", inicialmente para verificar la normalidad de las notas finales en los semestres donde se usaron los grupos de control y experimental (S2 y S3) se usó el test de Kolmogorov-Smirnov. Para evaluar las diferencias entre las medias en cada grupo en los semestres donde la distribución de notas fue normal, se utilizó el test paramétrico 
t-Student. Cuando las notas no siguen una distribución normal, se utiliza el test no paramétrico para muestras independientes UMann-Whitney y la prueba de la mediana para k muestras. Por último, para evaluar en los grupos gamificados posibles diferencias significativas, se aplicó la prueba Anova de un factor de Kruskal-Wallis y el test de las medianas para k muestras.

Se fijó un intervalo de confianza del 95,0\% para todos los casos, la significación estadística se interpretó según el siguiente criterio:

- $\quad$ Si p > 0,05 no existen diferencias significativas.

- $\quad$ Si $\mathrm{p} \leq 0,05$ existen diferencias significativas.

\section{Resultados}

A continuación, se describen las estrategias de gamificación para el desarrollo de tareas autónomas implementadas en Moodle para fortalecer el aprendizaje de P-I. Luego se exponen los resultados del análisis cuantitativo sobre los puntos de experiencia, el número de insignias ganadas por el cumplimiento de tareas autónomas, y las notas finales; y del análisis cualitativo de las encuestas realizadas a los estudiantes sobre las estrategias de gamificación y la plataforma Moodle gamificada; para al final realizar las pruebas de hipótesis correspondientes sobre la variable "rendimiento académico", tomando en cuenta las notas finales comparando los grupos gamificado (experimental) y normal (control).

\subsection{Metodología gamificada}

El diseño de la experiencia gamificada se realizó con el docente de la asignatura y se tomó en cuenta la problemática de falta de motivación por la realización de tareas autónomas (Beltrán, Sánchez, \& Rico, Análisis cuantitativo y cualitativo del aprendizaje de Programación I en la Universidad Central del Ecuador, 2015), se basó en los seis pasos planteados en el framework D6 (Werbach \& Hunter, 2012) adaptándolos al aprendizaje de programación:

- Definir los objetivos del curso: Se busca aumentar los conocimietos y capacidades de los estudiantes de P-I y mejorar su rendimiento académico.

- Diseñar las conductas objetivo: Se desea que los estudiants incrementen la motivación y compromiso en el desarrollo de las tareas de programación fuera del aula desarrollando el autoaprendizaje.

- Describir a los jugadores-estudiantes: Los jugadores serán los estudiantes de P-I de la FICA de la UCE. En general no concen de programación y no les gusta realizar tareas fuera del aula (Beltrán, Sánchez, \& Rico, Análisis cuantitativo y cualitativo del aprendizaje de Programación I en la Universidad Central del Ecuador, 2015).

- Elaborar los bucles de actividad: La experiencia gamificada se ambienta en un juego de ordenador de la Edad Media. El docente es el Sabio de la localidad, los alumnos serán aprendices de magos de la "Magia de la Programación”, los 
materiales de programación son fórmulas y recetas de magia, las tareas como ejercicios y pruebas son las actividades de hechicería, los programas Java son hechizos o conjuros, y los foros y chats son tabernas virtuales donde los aprendices podrán interactuar y ayudarse mutuamente.

La misión y reto consiste en viajar y alcanzar los diferentes niveles de la "Programación Mágica” como se muestra en la Figura 2.

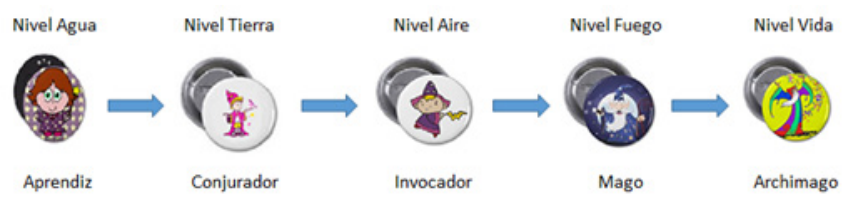

Figura 2 - Niveles de Programación Mágica. Fuente: elaboración propia

En cada nivel los alumnos deben realizar actividades en las que ganan puntos de experiencia y obtienen insignias que muestren su avance dentro de la plataforma, además de ser una recompensa por su esfuerzo. Cada nivel tiene mayor complejidad que el anterior, para que de esta manera el reto siga creciendo y motivando al aprendiz a superarlo. Para pasar de un nivel al siguiente, se necesita de la realización correcta de un número mínimo de tareas, o en caso contrario una vez transcurrido el tiempo límite para su realización se pasa de nivel sin acumular esos puntos. Además, podrá conseguir premios virtuales por la consecución de algunas competencias o cuando el sabio lo decida, como libros de magia de programación.

El estudiante podrá identificarse con un avatar en cada nivel. Se tendrá retroalimentación inmediata en cada tarea, el alumno podrá intentar las veces que sean necesarias para cumplir con ellas mientras el periodo de tiempo esté disponible. Para colaborar entre aprendices se tendrá una taberna virtual (foros).

Para motivar la competencia entre aprendices se tendrá un tablero donde verán los puntos de experiencia ganados. Además, los alumnos podrán ver gráficamente el avance que tienen y lo que les falta por cumplir para terminar cada nivel y sub nivel.

Los puntos de experiencia que ganen se transformarán en puntos de tareas autónomas para su nota final (50\% de la nota), el 50\% restante corresponde al examen final.

En la Tabla 2 se muestran los capítulos de Fundamentos de Java, niveles avatares, sub capítulos, sub niveles y puntos de experiencia por ganar.

- $\quad$ No se olvide de la diversión: Se busca motivación intrínseca cuando el aprendiz termine cada tarea de programación dentro un ambiente divertido, social y fácil de usar para jóvenes que están al día con la tecnología.

- Implementar las herramientas apropiadas: La plataforma gamificada será implementada en Moodle donde se podrá programar en Java. 


\begin{tabular}{|c|c|c|c|c|c|}
\hline Capítulo & Sub capítulo & Nivel & Avatar & Sub nivel & Puntos \\
\hline $\begin{array}{l}\text { Introducción a } \\
\text { la } P O O\end{array}$ & Introducción a la POO & Agua & Aprendiz & $\begin{array}{l}\text { Introducción a } \\
\text { la POO }\end{array}$ & 120 \\
\hline \multirow{6}{*}{$\begin{array}{l}\text { Fundamentos } \\
\text { de Java }\end{array}$} & Estructuras secuenciales & \multirow{6}{*}{ Tierra } & \multirow{6}{*}{ Conjurador } & Secuenciales & 120 \\
\hline & Estructura selectiva: if-else & & & if-else & 100 \\
\hline & Estructura selectiva: switch & & & Switch & 50 \\
\hline & Estructura repetitiva: for & & & For & 50 \\
\hline & Estructura repetitiva: while & & & While & 50 \\
\hline & $\begin{array}{l}\text { Estructura repetitiva: do- } \\
\text { while }\end{array}$ & & & do-while & 50 \\
\hline \multirow{2}{*}{$\begin{array}{l}\text { Arreglos y } \\
\text { Matrices }\end{array}$} & Arreglos & \multirow{2}{*}{ Aire } & \multirow{2}{*}{ Invocador } & Arreglos & 120 \\
\hline & Matrices & & & Matrices & 100 \\
\hline \multirow[t]{2}{*}{ Métodos } & Métodos & Fuego & Mago & Métodos & 320 \\
\hline & & Vida & Archimago & & \\
\hline
\end{tabular}

Tabla 2 - Capítulos, Niveles, Avatar y Puntuación de Fundamentos de Java

\subsection{Implementación en Moodle}

Las estrategias de gamificación diseñadas para el desarrollo de tareas autónomas de Fundamentos de Java de la asignatura P-I, se implementaron en el entorno virtual Moodle creándose la plataforma "Moodle Gamificado" (http://www.aprendizajegamificado. com) (Figura 3) donde se implementaron las siguientes dinámicas, mecánicas y componentes de juego (Werbach \& Hunter, 2012):
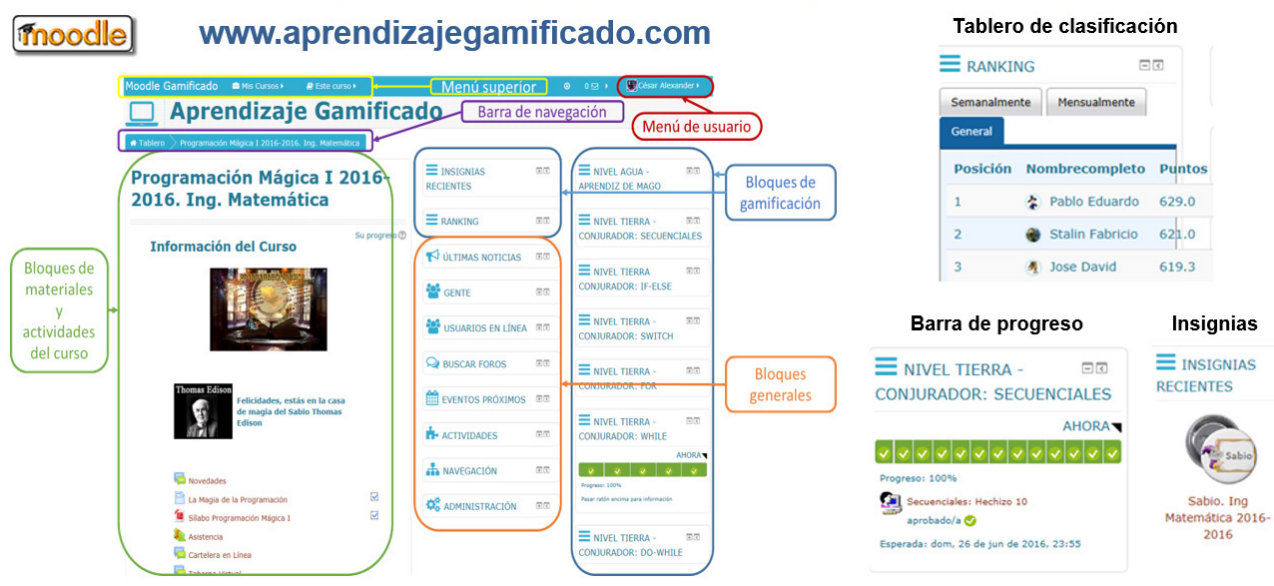

Figura 3 - Moodle Gamificado. Fuente: elaboración propia

- Dinámicas: Recompensas, estatus, competencia, logros, expresión e identidad propia, altruismo y ayuda, retroalimentación. 
- Mecánicas y componentes: Puntos, insignias, tabla de clasificaciones (plugin Ranking Block), barra de progreso (plugin Completion Progress), niveles, avatares, retroalimentación automática de código Java (plugin Virtual Programming Lab -VPL), colaboración, misiones y retos, bienes virtuales.

VPL (Thiébaut, 2015) permite codificar, compilar, ejecutar y evaluar automáticamente programas Java y otros lenguajes.

Las mecánicas y componentes se usan para conseguir las dinámicas, por ejemplo, los puntos e insignias indican recompensa y logro; los niveles significan logro, las tablas de clasificaciones muestran estatus y competencia, la barra de progreso da retroalimentación, etc. (Werbach \& Hunter, 2012).

\subsection{Análisis Cuantitativo}

Para medir los resultados del Aprendizaje Gamificado sobrela motivación y el compromiso de los estudiantes en el desarrollo de tareas autónomas y el rendimiento académico, se realizó un estudio con alcance explicativo, con un diseño cuasi-experimental con grupos experimental y de control en los S2 y S3, en el que se emplean grupos intactos de comparaciones ya existentes, no equivalentes. En los siguientes cursos, S4 a S7-2, se utiliza la metodología gamificada y se evalúan los resultados.

- Semestre 1: En este semestre, se podía ganar hasta 100 puntos de experiencia y 5 insignias. No existía límite de tiempo para terminar las tareas. Para ganar una insignia se necesitaba cumplir con el 100,0\% de las tareas, para vencer un nivel y pasar al siguiente sólo del 80,0\%.

Se observa que el 90,0\% de los estudiantes obtuvieron el nivel superior (VidaArchimago), significa que al menos realizaron correctamente el 80,0\% de las tareas en cada nivel. El 50,0\% tuvo 5 insignias, el 40,0\% ganaron 4, y sólo un alumno obtuvo 3 insignias. En cuanto a los puntos de experiencia, el 90,0\% obtuvo más de 88,90 que equivale a 8,89 en la nota de tareas autónomas.

- Semestre 2: La escala de puntos de experiencia pasó a un máximo de 1080 para tener mayor percepción de reconocimiento por las tareas que realizan, además se incrementó 9 insignias de sub nivel a las 5 de nivel que ya se tenía para premiar el cumplimiento de los hechizos de programación al cumplir cada sub nivel y no sólo al terminar un nivel. Al igual que el semestre anterior, no existía límite de tiempo para realizar todas las tareas. El 100,0\% de los estudiantes alcanzaron el nivel superior y ganaron el total de insignias.

- Semestre 3: En los $\mathrm{S} 1$ y S2, al no tener tiempo límite la realización de las tareas para que los aprendices avancen a su ritmo, ocasionaba que en algunos estudiantes las clases presenciales estaba por un capítulo o sub capítulo y las tareas autónomas por otro nivel o sub nivel. Al final, previo al examen, la mayoría se igualaba, en varios casos producto de copia. Por esta razón se puso tiempo límite para terminar o vencer los niveles y sub niveles que haga correspondencia con las clases presenciales, si un estudiante no terminó a tiempo las tareas definidas en el diseño, deja de ganar los puntos de experiencia y avatar de sub nivel correspondiente. 
De esta manera se incrementó el reto al tener tiempo máximo para vencer los niveles y sub niveles; además se influyó en la motivación, los alumnos intentaban una y otra vez los hechizos (ejercicios) y retos de hechicería (lecciones o pruebas) hasta vencerlos para no dejar de ganar los puntos e insignias, y finalmente para ir al mismo ritmo de las clases presenciales.

El 100,0\% de los estudiantes alcanzaron el nivel superior. El 81,3\% ganaron todas las insignias, lo que implica que hicieron todas las tareas autónomas en el tiempo establecido; el 12,5\% ganaron 13 insignias, el 3,1\% tuvo 11 insignias y el restante $9(3,1 \%)$.

El 90,6\% de los alumnos tuvieron más de 1000 puntos de experiencia que les da una nota final en tareas autónomas de 10,0; 2 estudiantes entre 900 y 1000 puntos (6,3\%), y sólo 1 menos de 900 (3,1\%).

- $\quad$ Semestre 4 a Semestre 7-2: En los siguientes semestres se usó la metodología óptima encontrada en el S3 y poder seguir validándola y aprendiendo de cada experiencia. El 88,0\% de los alumnos obtuvieron más de 900,01 puntos de experiencia y una nota superior a 9,01 en tareas autónomas.

El 84,8\% de los estudiantes obtuvieron el nivel de Vida-Archimago; el 67,4\% ganaron 14 insignias, es decir, hicieron todas tareas autónomas en el tiempo determinado, y el 25,0\% ganaron entre 9 y 13 insignias.

Antes de la implementación de la propuesta, la motivación no se mantenía constante durante el curso, disminuye mientras la complejidad de los ejercicios aumenta. Los resultados en todos los semestres muestran que se logró influir en los estudiantes por la realización de las tareas planificadas de programación en Moodle Gamificado, desarrollando y manteniendo una motivación intrínseca por el aprendizaje.

- Notas finales P-I: Para determinar si el Aprendizaje Gamificado tiene impacto en el rendimiento académico; se analizó el porcentaje de estudiantes aprobados, no aprobados (terminaron el curso sin aprobarlo), retirados (no terminaron el curso) y suspensos (no aprobados + retirados), comparando los resultados entre los grupos de control y experimental en los $\mathrm{S} 2$ y $\mathrm{S} 3$.

En la Tabla 3 se observa que el porcentaje de alumnos aprobados usando la metodología gamificada fue creciendo; en S1 aprobaron el 35,3\%, en S2 el $38,5 \%$, y en S3 el 97,0\%. Además, se identifica que se retiran menos, ya que en $\mathrm{S} 1$ se retiró el 41,2\%, en $\mathrm{S} 2$ un 23,1\% y en $\mathrm{S} 3$ sólo un 3,0\%.

\begin{tabular}{lccccc}
\hline Semestre & Metodología & Aprobados & No aprobados & Retirados & Suspensos \\
\hline$S 1$ & Gamificada & 35,3 & 23,5 & 41,2 & 64,7 \\
\hline$S 2$ & Normal & 33,3 & 16,7 & 50,0 & 66,7 \\
\hline$S 2$ & Gamificada & 38,5 & 38,5 & 23,1 & 61,5 \\
\hline$S 3$ & Normal & 77,4 & 12,9 & 9,7 & 22,6 \\
\hline$S 3$ & Gamificada & 97,0 & 0,0 & 3,0 & 3,0 \\
\hline
\end{tabular}

Tabla 3 - Notas finales (\%): Metodología Tradicional vs Gamificada 
Comparando las metodologías tradicional y gamificada, se observa que, en S2 el porcentaje de alumnos aprobados con la metodología gamificada fue del $38,5 \%$ y con la normal del 33,3\%; en el S3 la diferencia fue mayor, el 97,0\% aprobaron usando la metodología gamificada y el 77,4\% con la tradicional. La relación es contraria al analizar los estudiantes suspensos, quienes se retiraron y aquellos que no aprobaron.

Los mejores resultados se tuvieron en el $\mathrm{S}_{3}$ con el uso de las estrategias de gamificación en un entorno e-Learning donde se implementó las mejores prácticas de las experiencias adquiridas en los periodos anteriores; el 97,0\% aprobaron, sólo el 3,0\% se retiró; de quienes terminaron la asignatura, el 100,0\% aprobó. Esto permitió perfeccionar varios elementos en las estrategias de gamificación y su forma de implementarlas en Moodle, y encontrar de esta manera la propuesta óptima al problema planteado en la investigación sobre la baja motivación de los alumnos por la ejecución de las tareas que realizan fuera del aula.

El porcentaje de estudiantes que aprobaron, suspendieron, no aprobaron y se retiraron en los siguientes semestres donde se usó Aprendizaje Gamificado se detalla en la Tabla 4. El 79,4\% aprobó, el 18,0\% no aprobó y el 2,6\% se retiró, confirmando que la motivación por el aprendizaje de programación desarrollando tareas autónomas en Moodle Gamificado se mantuvo en todos los cursos debido al alto porcentaje de alumnos que aprobaron.

\begin{tabular}{lcccc}
\hline Semestre & Aprobados & No aprobados & Retirados & Suspensos \\
\hline Semestre 4 & 87,0 & 8,7 & 4,3 & 13,0 \\
\hline Semestre 5 & 85,7 & 0,0 & 14,3 & 14,3 \\
\hline Semestre 6-1 & 87,1 & 12,9 & 0,0 & 12,9 \\
\hline Semestre 6-2 & 86,7 & 8,9 & 4,4 & 13,3 \\
\hline Semestre 7-1 & 67,5 & 30,0 & 2,5 & 32,5 \\
\hline Semestre 7-2 & 72,1 & 27,9 & 0,0 & 27,9 \\
\hline Total & 79,4 & 18,0 & 2,6 & 20,6 \\
\hline
\end{tabular}

Tabla 4 - Notas finales (\%): Metodología Gamificada

\subsection{Análisis Cualitativo}

Al finalizar el curso se realizó a los alumnos una encuesta electrónica anónima para conocer su opinión sobre la experiencia al desarrollar las tareas autónomas en Moodle Gamificado y sobre la metodología gamificada, los resultados son los siguientes:

El uso de Moodle Gamificado implicó que el estudiante esté al pendiente día a día de las tareas que debía realizar. Obtener los puntos de experiencia, recompensas, insignias y las calificaciones es cuestión de esfuerzo y voluntad. La valoración de excelente y muy bueno fue del 85,9\% en los semestres estudiados.

El 90,2\% de los alumnos afirmaron que la metodología propuesta fue mucho mejor y mejor que la metodología tradicional. El 85,3\% de los estudiantes valoraron la 
metodología "Gamificación en el ambiente e-Learning" como excelente y muy buena. Los alumnos realizaban los ejercicios de programación una y otra vez hasta que conseguían que funcionen correctamente, no se daban por vencidos, de esta manera lograron desarrollar sus conocimientos en Java.

El 87,7\% de los estudiantes opinaron estar totalmente de acuerdo y de acuerdo que el aprendizaje de programación fue divertido. En esta nueva forma de aprender existía competencia y colaboración entre los estudiantes. Sin necesidad de una orden del profesor, los alumnos realizaban las tareas autónomas publicadas en la plataforma, el propósito era obtener mayor puntuación y premios para superar a sus compañeros.

El Aprendizaje Gamificado implementado en Moodle influyó en la motivación de los estudiantes, el 81,0\% calificaron su motivación como muy alta y alta. El 90,8\% de los alumnos afirmaron que su motivación fue mucho mejor y mejor que en el I H-S donde se usó la metodología tradicional. Son motivados por los premios, insignias, retroalimentación y otras dinámicas y mecánicas de juego que se aplican en el curso.

Las dinámicas mejor evaluadas fueron "los logros" y "las recompensas". Entre las mecánicas de juego más valoradas estuvieron "las misiones o retos", "puntos", "desbloqueo de contenidos" y "retroalimentación”.

Las dinámicas y mecánicas aplicadas en las estrategias de gamificación motivaron a los alumnos para aprender a programar, el 92,6\% quedaron satisfechos y muy satisfechos con las estrategias de gamificación aplicadas (Tabla 5).

\begin{tabular}{llllll}
\hline Semestre & $\begin{array}{l}\text { Muy } \\
\text { Satisfactorio }\end{array}$ & Satisfactorio & Indiferente & $\begin{array}{l}\text { Poco } \\
\text { Satisfactorio }\end{array}$ & Malo \\
\hline$S_{1}$ & 66,7 & 33,3 & 0,0 & 0,0 & 0,0 \\
\hline$S 2$ & 72,7 & 27,3 & 0,0 & 0,0 & 0,0 \\
\hline$S_{3}$ & 53,3 & 43,3 & 3,3 & 0,0 & 0,0 \\
\hline$S 4$ & 63,2 & 36,8 & 0,0 & 0,0 & 0,0 \\
\hline$S 5$ & 100,0 & 0,0 & 0,0 & 0,0 & 0,0 \\
\hline$S 6$ & 43,2 & 54,5 & 2,3 & 0,0 & 0,0 \\
\hline$S 7$ & 20,5 & 56,8 & 18,2 & 4,5 & 0,0 \\
\hline Total & 46,6 & 46,0 & 6,1 & 1,2 & 0,0 \\
\hline
\end{tabular}

Tabla 5 - Porcentaje de satisfacción del Curso Gamificado

\subsection{Comprobación de la hipótesis}

Para determinar estadísticamente que la propuesta permitió mejorar en el rendimiento académico de los estudiantes de P-I, se realizó la comprobación de la hipótesis alternativa: "El rendimiento académico de los estudiantes en la asignatura P-I de la FICA de la UCE es significativamente diferente entre los estudiantes que emplean la propuesta y aquellos que no la siguen".

En el S2 se evaluaron mediante la prueba t-Student (por ser una distribución normal, Tabla 6) la diferencia entre las notas finales para los grupos que estudiaron con 
la metodología tradicional (control) y la metodología gamificada (experimental), mostrando que no existen diferencias entre ambos grupos pues p>0,05 (Tabla 7).

\begin{tabular}{lcc}
\hline \multirow{2}{*}{ Semestres } & \multicolumn{2}{c}{ Z de Kolmogorov-Smirnov } \\
\cline { 2 - 3 } & Estadístico de prueba & p (sig. Asintótica bilateral) \\
\hline$S 1$ & 0,223 & 0,025 \\
\hline$S 2$ & 0,112 & 0,200 \\
\hline$S 3$ & 0,263 & 0,000 \\
\hline
\end{tabular}

Tabla 6 - Test de Normalidad de Kolmogorov-Smirnov

\begin{tabular}{|c|c|c|c|c|}
\hline \multirow[b]{2}{*}{ Metodología } & \multicolumn{4}{|c|}{ Test t-Student } \\
\hline & $\overline{\mathbf{X}} \pm$ D.E & $t$ & $\mathbf{p}$ & IC 95\% \\
\hline Normal & $18,45 \pm 7,71$ & \multirow{2}{*}{1,057} & \multirow{2}{*}{0,076} & \multirow{2}{*}[-0,67;12,46]{} \\
\hline Gamificada & $24,35 \pm 8,13$ & & & \\
\hline
\end{tabular}

Tabla 7 - Test T-Student Notas Semestre 2

Luego, en el S3, donde se usaron las mejores prácticas encontradas de acuerdo a las experiencias tenidas en los semestres anteriores, se evaluó la diferencia de las notas finales entre los grupos bajo ambas metodologías. En este caso, al no ser normal la distribución de la variable (Tabla 6), se aplicó el test no paramétrico para muestras independientes UMann-Whitney. Al aplicar la prueba se comprueba la diferencia de los dos grupos $(\mathrm{p}<0,05)$, por lo que se rechaza la hipótesis nula "El rendimiento académico de los estudiantes en la asignatura de P-I de la FICA de la UCE no es significativamente diferente entre los estudiantes que emplean la propuesta y aquellos que no la siguen" en favor de la hipótesis alternativa descrita anteriormente, es decir, podemos afirmar que existe una diferencia significativa entre las notas del grupo bajo la estrategia de aprendizaje tradicional y las notas del grupo bajo la estrategia gamificada (Tabla 8).

\begin{tabular}{lll}
\hline \multicolumn{1}{c}{ Estadígrafos y test utilizados } & \multicolumn{2}{l}{ Metodología de enseñanza } \\
\cline { 2 - 3 } & Normal & Gamificada \\
\hline $\bar{X}_{ \pm} D . E$ & $26,88 \pm 7,9$ & $32,58 \pm 5,0$ \\
\hline Mediana & 29,0 & 32,8 \\
\hline UMann-Whitney & 740,500 & \\
\hline Estadístico estandarizado & $-3,587$ & \\
\hline Significación asintótica bilateral $(p)$ & 0,000 & \\
\hline Mediana de grupos & 30,615 & \\
\hline Test con corrección de Yates (Chi cuadrado) & 12,262 & \\
\hline Significación asintótica bilateral $(p)$ & 0,000 & \\
\hline
\end{tabular}

Tabla 8 - Test No Paramétrico Notas Semestre 3 
Finalmente, al aplicar el test de Kruskal-Wallis y el test de medianas para k muestras a los semestres donde se usó el Aprendizaje Gamificado, se verifica que existe una diferencia de grupos ( $<<0,05)$, por lo tanto, los resultados de las notas finales bajo esta metodología fueron diferentes en los semestres y cursos donde fue aplicada (Tabla 9). Además, como el promedio de las notas $(28,65)$ es mayor que el mínimo para aprobar el curso (27,50), se comprueba que existió un buen rendimiento académico en los cursos donde se aplicó la propuesta.

\begin{tabular}{ll}
\hline Estadígrafos y test utilizados & Notas cursos gamificados \\
\hline $\bar{X}_{ \pm} D . E$ & $28.65 \pm 7.2$ \\
\hline $\begin{array}{l}\text { Anova de 1-factor de Kruskal-Wallis: Significación asintótica } \\
\text { bilateral (p) }\end{array}$ & 0.000 \\
\hline Mediana de grupos & 30.660 \\
\hline Mediana de grupos: Significación asintótica bilateral $(p)$ & 0.000 \\
\hline
\end{tabular}

Tabla 9 - Test No Paramétrico Notas cursos gamificados

\section{Conclusiones}

La presente investigación parte de conceptos establecidos para cambiar una realidad difícil, los estudiantes de programación no tienen suficiente motivación por el aprendizaje, pues las clases pueden llegar a ser monótonas o muy tediosas, y la falta de gusto por realizar tareas fuera del aula (Yacob, Yazid, \& Saman, 2012); se asume entonces la necesidad del uso de estrategias metodológicas y tecnológicas con la finalidad de aumentar la motivación, a partir de la introducción de la gamificación en el desarrollo del aprendizaje, de combinar la diversión con el aprender (Kyriakova, Angelova, \& Yordanova, 2014).

La implementación de gamificación como apoyo en el aprendizaje de programación debe realizarse en un ambiente e-Learning (Beltrán, Sánchez, \& Rico, Incrementar la motivación en el aprendizaje de Fundamentos de Programación Java usando Moodle Gamificado, 2016) (Cacho \& Rodrigo, 2014), pues ayuda en el incremento de la motivación de los estudiantes, la colaboración, participación, trabajo en equipo y compromiso (Machuca-Villegas, Gasca-Hurtado, Restrepo, \& Puente, 2020) (GascaHurtado, Gómez-Álvarez, \& Manrique-Losada, 2019), factores condicionantes decisivos del aprendizaje y el rendimiento académico; además influye en la mejora de su rendimiento académico, principalmente en la participación de las tareas en ambientes virtuales y en la asistencia, y en menor medida en las calificaciones (Muntean, 2011) (Simões, Díaz, \& Fernández, 2013).

La presente investigación tiene como objetivo principal presentar una propuesta metodológica, "Aprendizaje Gamificado", y tecnológica, "Moodle Gamificado", para el desarrollo de tareas autónomas en la asignatura P-I, que ayuden a promover el aprendizaje e incentivar a los alumnos por la programación en un ambiente tecnológico y virtual.

Los resultados del análisis cualitativo muestran que se desarrolló en los estudiantes un interés genuino por la programación creándose una motivación intrínseca que los 
motiva a estudiar y realizar las actividades planificadas, para de esta forma adquirir el mayor nivel de conocimientos. El uso del Aprendizaje y Moodle Gamificado influyó en la motivación de los alumnos por la ejecución de las tareas autónomas, y fue fundamental para el cumplimiento de las mismas pues se divertían haciéndolas.

De los análisis cualitativo y cuantitativo, y pruebas de hipótesis realizadas; se concluye que la implementación de estrategias de gamificación sobre una plataforma virtual de aprendizaje, no sólo influyó en la motivación de los alumnos para desarrollar tareas autónomas, sino que también contribuyó a tener mejores calificaciones mejorando así su rendimiento académico, en la asignatura P-I de la FICA de la UCE. Es importante notar que el docente tiene un papel fundamental en la aplicación de la metodología, debe ir adaptándola y mejorándola, pues cada grupo de alumnos es diferente y tiene sus propias capacidades.

Como trabajo futuro se propone aplicar el Aprendizaje Gamificado en todos los cursos de Programación I y en asignaturas relacionadas (como Programación II o Estructura de Datos). Se plantea también aplicarlo y adaptarlo en otras asignaturas que se imparten en las diversas áreas de conocimiento en la universidad haciendo uso de Moodle Gamificado $\mathrm{u}$ otra plataforma virtual, permitiendo observar el comportamiento y resultados de la aplicación determinando las mejores estrategias y herramientas que permitan mejorar la motivación y el rendimiento académico de los alumnos. Para esto es importante desarrollar planes de actualización docente en el uso de las TIC, y en nuevas metodologías que favorezcan el proceso de enseñanza-aprendizaje.

\section{Referencias}

Beltrán, J., Sánchez, H., \& Rico, M. (2015). Análisis cuantitativo y cualitativo del aprendizaje de Programación I en la Universidad Central del Ecuador. Revista Tecnológica ESPOL - RTE, 28(5), 194-210. http://bit.ly/2NllYoL

Beltrán, J., Sánchez, H., \& Rico, M. (2016). Incrementar la motivación en el aprendizaje de Fundamentos de Programación Java usando Moodle Gamificado. 11th Iberian Conference on Information Systems and Technologies (págs. 1189-1193). Gran Canaria, España: IEEE. doi:https://doi.org/10.1109/CISTI.2016.7521419

Cacho, G., \& Rodrigo, M. (2014). Gamifying Moodle: Integrating Game Elements Into an Open-Source Learning Management System. In International Symposium on Computing for Education, (págs. 22-29). Borocay, Philippines. http://bit.ly/2Hho5iu

Fuentes-Rosado, J., \& Moo-Medina, M. (2017). Dificultades de aprender a programar. Revista educacion en ingenieria, 12(24), 76-82. doi:https://doi.org/10.26507/rei. v12n24.728

Gasca-Hurtado, P., Gómez-Álvarez, C., \& Manrique-Losada, B. (2019). Using Gamification in Software Engineering Teaching: Study Case for Software Design. World Conference on Information Systems and Technologies, 932, 244-255. https://link.springer.com/chapter/10.1007/978-3-030-16187-3_24 
González, C, \& Mora, A. (2015). Técnicas de gamificación aplicadas en la docencia de Ingeniería Informática. Revista de Investigación en Docencia Universitaria de la Informática, 8(1), 29-40. http://bit.ly/2TOLk8i

Guo, M., Chai, T., \& Qian, K. (2010). Design of Online Runtime and Testing Environment for Instant Java Programming Assessment. Proc. Information Technology, 1102-1106.

Ibáñez, M., Di-Serio, A., \& Delgado-Kloos, C. (2014). Gamification for engaging Computer Science Students in Learning Activities. IEEE Transactions on learning technologies, 7(3), 291-301. doi:https://doi.org/10.1109/TLT.2014.2329293

Jenkins, T. (2001). The motivation of students of programming. ITiCSE 2001 6th annual conference on Innovation and technology in computer science education (págs. 53-56). Canterbury, UK: University of Kent at Cantebury. doi:https://doi.org/10.1145/377435.377472

Kyriakova, G., Angelova, N., \& Yordanova, L. (2014). Gamification in education. 9th International Balkan Education and Science Conference. Edirne, Turkey. http://bit.ly/2ZcIbR3

Lorenzo, C., \& Buendía, M. (2016). Uso de la Web Social en Enseñanzas Medias. Interciencia, 198-203. http://bit.ly/2ZhG2Ya

Machuca-Villegas, L., Gasca-Hurtado, G., Restrepo, L., \& Puente, S. (2020). Elementos de Gamificación en el Contexto de Ingeniería de Software. RISTI - Revista Ibérica de Sistemas e Tecnologias de Informação (E27), 718-732.

Mas, C., \& Medinas, M. (2007). Motivaciones para el estudio en universitarios. Anales de Psicología, 23(1), 17-24. http://bit.ly/2ZcNeAN

Muntean, C. (2011). Raising engagement in e-learning through gamification. 6th International Conference on Virtual Learning ICVL, (págs. 323-329). Romania. http://bit.ly/30movIP

Oviedo, M., \& Ortiz Uribe, F. (2012). La enseñanza de la programación. México DF: Academias de Humanidades de la UPIICSA.

Pérez, A., \& Almela, J. (2018). Gamificación transmedia para la divulgación científica y el fomento de vocaciones procientíficas en adolescentes. Comunicar, XXVI(55), 93-103. doi:https://doi.org/10.3916/C55-2018-09

Schefer-Wenzl, S., \& Miladinovic, I. (2018). Teaching Software Engineering with Gamification Elements. International Journal of Advanced Corporte Learning, 11(1), 48-51. doi:https://doi.org/10.3991/ijac.v11i1.9169

Serna, E., Mauricio, M., San Miguel, T., \& Megías, J. (2016). Experiencia de gamificación en Docencia Universitaria: aprendizaje activo y entretenido. In-Red 2016 - II Congreso nacional de innovación educativa y docencia en red. Valencia, España: Editorial Universitat Politècnica de València. doi:https://doi.org/10.4995/ INRED2016.2016.4292 
Simões, J., Díaz, R., \& Fernández, A. (2013). A social gamificaction framework for a K-6 learning platform. Computer in Human Behavior, 29(2), 345-353. doi:https://doi.org/10.1016/j.chb.2012.06.007

Tejedor, F. (2000). El diseño y los diseños en la evaluación de programas. Revista de Investigación Educativa, 18(2), 319-339. http://bit.ly/2MluwCB

Thiébaut, D. (2015). Automatic evaluation of computer programs using Moodle's virtual programming lab (VPL) plug-in. Journal of Computing Sciences in Colleges, 30(6), 145-151. http://bit.ly/32bO8Qa

Urh, M., Vukovic, G., Jereb, E., \& Pintar, R. (2015). The Model for Introduction of Gamification into E-learning in Higher Education. 7th World Conference on Educational Sciences, 197, págs. 388-397. Kranj, Slovenia. doi:https://doi.org/10.1016/j.sbspro.2015.07.154

Watson, C., Li, F., \& Lau, R. (2011). Learning programming languages through corrective feedback and concept visualisation. Advances in Web-Based Learning - ICWL 2011 (págs. 11-20). Hong Kong: Springer. doi:https://doi.org/10.1007/978-3-64225813-8_2

Werbach, K., \& Hunter, D. (2012). Level 5: Game Changer: Six Steps to Gamification. En K. Werbach, \& D. Hunter. For the Win: How Game Thinking Can Revolutionize Your Business (págs. 85-102). Philadelphia: Wharton Digital Press. http://bit.ly/2zigrjl

Yacob, A., Yazid, M., \& Saman, M. (2012). Assessing level of motivation in learning programming among engineering students. TATI University, (págs. 425-432). Kemaman. https://bit.ly/2HhDGi1 